\title{
Primary Left Atrial Angiosarcoma Presenting as Acute Coronary Syndrome
}

\author{
Sandra Jaksic Jurinjak, MD,PhD, ${ }^{1}$ Josip Vincelj, MD,PhD,${ }^{1}$ Igor Rudez, MD,PhD, ${ }^{2}$ Ante Lisicic, MD, ${ }^{1}$ \\ Ivana Jurin, $\mathrm{MD},{ }^{1}$ Spomenka Manojlovic, $\mathrm{MD}, \mathrm{PhD},{ }^{3}$ Mario Udovicic, $\mathrm{MD}, \mathrm{PhD},{ }^{1}$ Boris Starcevic, $\mathrm{MD}, \mathrm{PhD}^{1}$ \\ ${ }^{1}$ Department of Cardiovascular Diseases, University Hospital Dubrava, Avenija Gojka Šuška 6, 10000 Zagreb, Croatia; ${ }^{2}$ Department \\ of Cardiac and Transplantation Surgery, University Hospital Dubrava, Avenija Gojka Šuška 6, 10000 Zagreb, Croatia; ${ }^{3}$ Department of \\ Pathology, University Hospital Dubrava, Avenija Gojka Šuška 6, 10000 Zagreb, Croatia
}

\section{ABSTRACT}

Primary cardiac neoplasms are extremely rare and often overlooked as differential diagnosis. Angiosarcomas are the most common primary malignant neoplasms of the heart often with nonspecific symptoms. We present a 43 -year-old woman admitted to our hospital with chest pain and inferoposterolateral myocardial infarction. Coronary angiography indicated the distal occlusion of the left circumflex artery. Transthoracic and transoesophagic echocardiography revealed a mass in the left atrium with probable myocardial infiltration and vascularisation. The mass in the left atrium was removed by surgical resection, and histopathology confirmed angiosarcoma. We emphasize the pivotal role of transthoracic and transoesophageal echocardiography in evaluating even rare differential diagnosis of acute coronary syndrome as cardiac neoplasms.

\section{INTRODUCTION}

Primary cardiac tumors are rare, constituting about one out of every 500 cardiovascular surgical cases, most of which are benign. Primary cardiac angiosarcomas are the most common histological subtype of malignant tumors and constitute about 30 percent of the primary cardiac malignant cases [Blackmon 2009; Ferguson 2002]. Incidence at autopsy is from 0.0001 percent to 0.0003 percent, mostly located in the atrium [Blackmon 2009]. Overall, the prognosis of patients with primary cardiac angiosarcoma is very poor. Surgical resection in combination with chemotherapy and radiation is recommended as soon as diagnosis is made. We describe the case of a young woman in which neoplasm of the left atrium, namely angiosarcoma, was suspected by echocardiography in acute coronary syndrome.

Case report: A 43-year-old woman with coronary risk factors, obesity, and smoking was admitted to our hospital with chest pain, electrocardiographic (ECG) changes on admission, and elevated cardiac troponin I, which suggested acute coronary syndrome (ACS). She had no previous chest pain

Received fanuary 9, 2019; accepted February 13, 2019.

Correspondence: Sandra Faksic furinjak, MD,PhD, University Hospital Dubrava, Avenija Gojka Šuška 6, 10000 Zagreb, Croatia (email: sjaksicj@ gmail.com). or dyspnoea on exertion. ECG demonstrated a sinus rhythm (80/beats/minute) and ST segment depression of approximately $2 \mathrm{~mm}$ in leads $\mathrm{V} 1$ to $\mathrm{V} 3$ and elevation in inferior and lateral leads (Figure 1).

Her cardiac markers were elevated (troponin I: $4.6 \mathrm{ng} / \mathrm{L}$ ) and creatine kinase peak $1517 \mathrm{U} / \mathrm{L}$. The patient's blood pressure was 148/95 mmHg; her heart rate was 80 beats/min; her oxygen saturation was 97 percent with room air; and her respiratory rate was 14 breaths/min. A chest X-ray done prior to attempt of PCI was normal. Acute inferoposterolateral myocardial infarction was suspected, so emergency coronary angiography was performed, indicating distal occlusion of left circumflex artery $(\mathrm{ACx})$ with poor flow after multiple dilatations but with symptoms and electrocardiographic changes normalization. No atherosclerotic plaque of coronary arteries was found. Percutaneous coronary interventions were performed without aspiration for coronary thrombus because the culprit lesion was too distal.

Real time three-dimensional transthoracic echocardiography (3D TTE) followed, revealing a cardiac mass in the lateral wall of the left atrium. This is best seen in apical 4 chamber view (Figure 2, A). Transthoracic echocardiography showed normal left ventricular (LV) systolic function with LV ejection fraction about 55 percent and no regional wall motion dysfunction. The left atrium (LA) and LV, as well as other heart chambers, were normal in size. Transoesophageal echocardiography (TEE) was done, showing locally

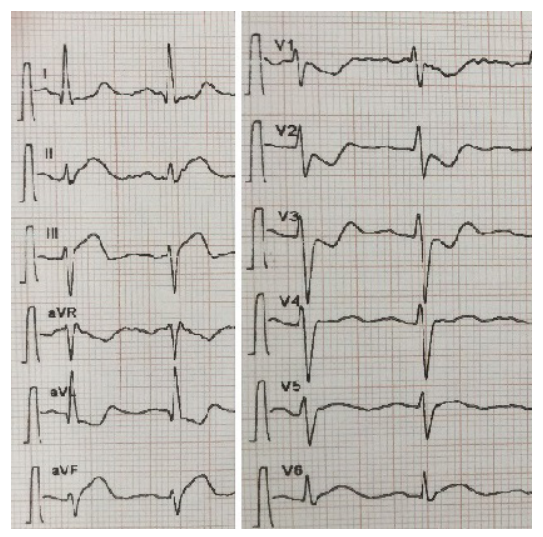

Figure 1. Electrocardiogram was recorded on admission demonstrating ST segment depression of approximately $2 \mathrm{~mm}$ in leads V1 to V3, and elevation in inferior and lateral leads. 

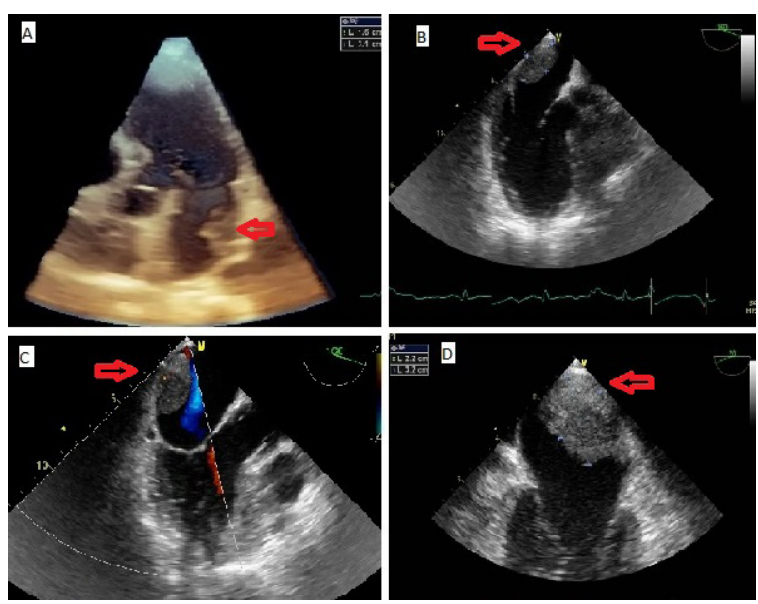

Figure 2. A, Transthoracic echocardiography 3D showing cardiac neoplasm in left atrium B, C, D, Transesophageal echocardiography showing a mass in the left atrium with vascularisation (midoesophageal long axis view-160 degrees combined with Doppler, 2-chamber view with zoom- 25 degrees, respectively).
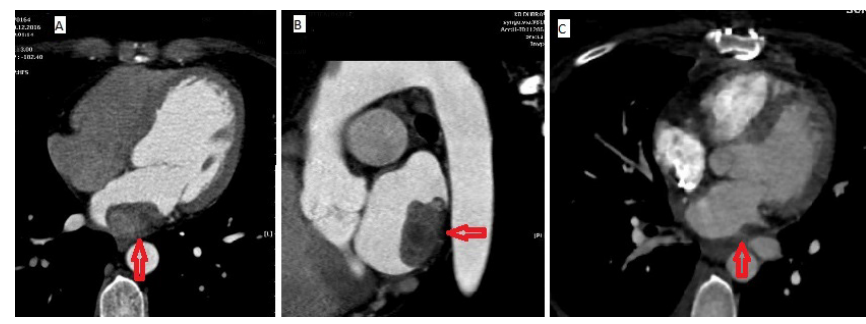

Figure 3. A, B, Thoracic computed tomography has confirmed the mass in the left atrium. C, Thoracic computed tomography after tumor was successfully resected in toto, showing no mass in the left atrium.

advanced cardiac mass in the posterolateral wall of left atrium partially infiltrating myocardium toward the pulmonary vein, size of $3.2 \mathrm{~cm} \times 2.2 \mathrm{~cm}$ with probable vascularisation suggesting angiosarcoma (Figure 2, B, C, D). The tumor was not infiltrating related structures, although it was near a mitral valve, which was proven to be normal functioning.

Thoracic computerized tomography (CT) confirmed the diagnosis of a vascularised cardiac mass, $38 \times 35 \times 20 \mathrm{~mm}$, in the left atrium with no metastasis or infiltrating other parts of heart (Figure 3, A, B). CT also was used to check for systemic metastases, which were not confirmed (Figure 3).

After median sternotomy, standard aortic and bicaval cannulation was performed, the heart was arrested with cold blood cardioplegia and the left atrial (LA) mass was approached through the interatrial groove. The tumor mass was inspected and successfully resected in toto as it was not infiltrating local structures (Figure 4, A, B). A small defect in the LA posterior wall was sutured with 4-0 polypropylene suture. The circumflex artery was not addressed because it was considered to be compressed by the tumor mass. The LA appendage was left intact as there were no signs of a clot, and the patient was in sinus rhythm. The mitral valve was not affected by the tumor, and after resection was competent on
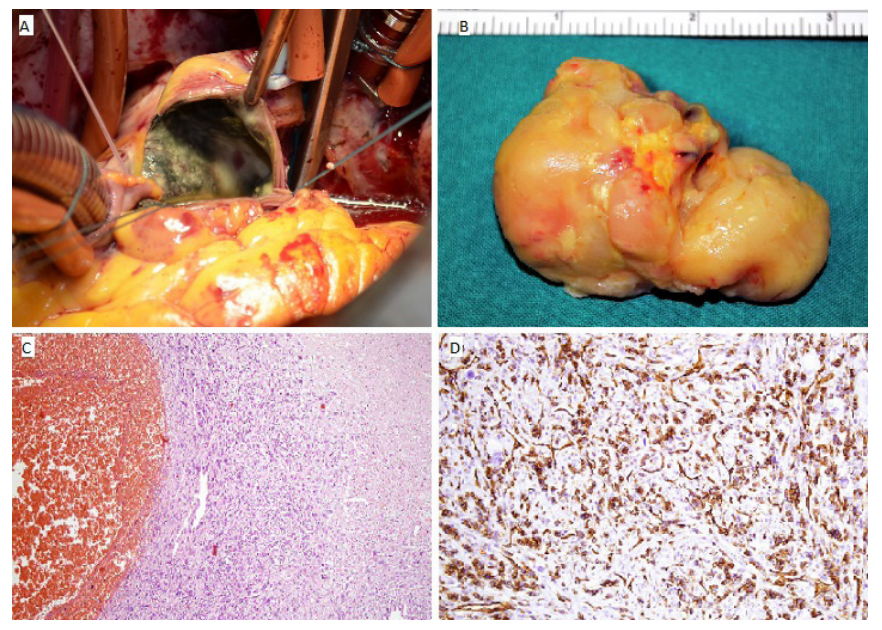

Figure 4. A, Neoplastic mass in the left atrium, intraoperative situs with view through the interatrial groove. B, Left atrial mass after surgical resection. Histological finding. C, Solid sheets of atypical endothelial cells with anastomosing irregular vascular channels and a dilated haemorrhagic pool (HE x 100). D, Immunohistochemical positivity with endothelial marker CD31.

a water test, which was later performed during intraoperative TEE. The patient was successfully weaned from cardiopulmonary bypass and without any inotropic support transferred into the ICU. A contingency plan, in case the tumor proved to be too large to be resected in toto, was to explant the heart, resect the LA mass, and then to re-implant the heart. After tumor resection, the remaining defect in the LA would have been taken care of with a bovine pericardial patch. Histopathologic findings confirmed the diagnosis of primary left atrial angiosarcoma (Figure 4, C, D). The patient was referred to the oncology department for chemotherapeutic and radiotherapeutic interventions.

\section{DISCUSSION}

Primary cardiac sarcomas have reported incidence of 0.0001 percent in collected autopsy series, reflecting the small percentage of body weight of heart ( 0.5 percent) compared with peripheral muscle (40 percent) [Silverman 1980; Talbot 2002]. Clinical diagnosis of angiosarcoma is difficult due to non-specific symptoms so detecting malignancy at an early stage is challenging [Engelen 2005]. Angiosarcomas are aggressive malignant neoplasms that most commonly occur in the head and neck, skin, breast, liver, spleen, and deep tissue with high rate of local recurrence and systemic metastases [Sturgis 2003].

Most common symptoms of primary cardiac sarcoma include chest pain, myalgia, palpitation, dyspnoea, or clinical manifestations as pericardial effusion or tamponade, vena cava obstruction, arrhythmia and chronic heart failure, presenting mostly as right-sided heart failure because malignant tumors are found mainly in the right heart. Most common site of primary cardiac angiosarcoma is the right atrium, occurring 
between the third and fifth decade of life with male predominance [Holloway 2009]. Cardiac sarcomas generally have a poor prognosis with a median survival of only 6 months due to 80 percent of patients presenting with distant metastasis at diagnosis [Herrmann 1992; Movsas 1998].

Preferred method of treatment is surgery in combination with chemotherapy and radiation because of the high probability of metastases [Oh 2013].

The exact benefits of chemotherapy and radiation are still being investigated [Bakaeen 2003]. Combined modality approach has been reported to be successful in few cases [Holloway 2009]. Complete resection has survival of 24 months compared with 10 months in those with incomplete resection [Putnam 1991].

Orthotropic heart transplantation has been performed in selected patients, but there is a high risk of tumor recurrence, metastasis or enhancement of tumor growth by immunosuppressive drugs [Crespo 1993; Michler 1997].

In our case, a cardiac mass with probable myocardial infiltration and vascularisation was observed by real time $3 \mathrm{D}$ TTE and confirmed with TEE. TEE in acute setting is indicated to better define the extension of cardiac neoplasm and has a higher resolution $(1 \mathrm{~mm}-3 \mathrm{~mm})$ and therefore can detect possible vascularisation, as in our case. Main differential diagnosis was myxoma as most common benign tumor located in the left atrium. Other differential diagnosis of left atrial mass includes thrombus and malignant causes such as metastatic involvement of the heart or other sarcomas and lymphoma [Holloway 2009].

On CT, angiosarcomas appear as irregular lobulated lowattenuation masses, but CT is also used to detect potential metastatic involvement [Holloway 2009].

Angiosarcoma of the left atrium in our patient was confirmed after surgical excision and histopathologic findings (Figure 3, C, D). Recommendations for the treatment of nonmetastatic cardiac sarcoma include exploration for local control of the primary tumor, removal of possible obstructive symptoms, and to prolong disease-free survival [Baay 1994].

Cardiac tumor, namely primary angiosarcoma can present as ACS, as in our patient. Primary cardiac angiosarcoma of the left atrium presenting as acute coronary syndrome has been reported in a scarce number of cases to the best of our knowledge [Duyuler 2015; Kurian 2006].

Symptoms of myocardial ischemia and elevated cardiac biomarkers can be result of myocardial involvement or coronary artery invasion [Duyuler 2015]. Our patient presented with ACS, possibly due to the setting of the related structures, perhaps from compression of the circumflex coronary from the mass effect of the tumor or thromboembolism originating from the surface of the tumor and not in situ cloth. Although prognosis of cardiac angiosarcoma is generally poor due to high frequency of metastatic spread, early detection may lead to a more favourable outcome.

Advanced echocardiographic methods (real time 3D echocardiography) and high resolution tomographic cardiac imaging (CT) have improved the diagnostic ability that could possibly improve prognosis in neoplasms with high metastatic potential [Kurian 2006]. Although it could still be difficult to detect malignancy, as a primary left atrial angiosarcoma at an early stage (as was in our case), transthoracic and transoesophageal echocardiography are pivotal imaging methods to evaluate rare differential diagnoses of ACS. This could help clinicians in search for malignant etiology and possibly help improve patient prognosis.

\section{REFERENCES}

Baay P, Karwande SV, Kushner JP, et al. 1994. Successful treatment of a cardiac angiosarcoma with combined modality therapy. J Heart Lung Transplant 13:923-925.

Bakaeen FG, Reardon MJ, Coselli JS, et al. 2003. Surgical outcome in 85 patients with primary cardiac tumors. Am J Surg 186: 641-47.

Blackmon SH, Reardon MJ. 2009. Surgical treatment of primary cardiac sarcomas. Tex Heart Ins J 36: 451-52.

Crespo MG, Pulpon LA, Pradas G, et al. 1993. Heart transplantation for cardiac angiosarcoma: should its indication be questioned? J Heart Lung Transplant 12:527-530.

Duyuler S, Demirkan B, Guray Y, et al. 2015. Echocardiography in differential diagnosis of chest pain and elevated cardiac biomarkers: a case of cardiac angiosarcoma. J Eur Echocariogr 12:406-407.

Engelen M, Bruch C, Hoffmeier A, et al. 2005. Primary left atrial angiosarcoma mimicking severe mitral valve stenosis, Heart 91:4- e27.

Ferguson ER, Walsh GL. 2002. Sarcomas of the heart and great vessels. In: Pollock, RE. Soft tissue sarcomas. Hamilton: CB Decker Inc. 155-56, 158-60.

Herrmann MA, Shankerman RA, Edwards WD, et al. 1992. Primary cardiac angiosarcoma: a clinicopathologic study of six cases. J Thorac Cardiovasc Surg 103:655-664.

Holloway BJ, Agarwal PP. 2009. AJR teaching file: right atrial mass in a woman with dyspnoea on exertion. AJR Am J Roentgenol 192: S49-52.

Kurian KC, Weisshaar D, Parekh H, et al. 2006. Primary cardiac angiosarcoma: case report and review of the literature. Cardiovascular Pathology 15:110-112.

Michler RE, Goldstein DJ. 1997. Treatment of cardiac tumors by orthotopic cardiac transplantation. Seminars in Oncology 24:534-539.

Movsas B, Teruya-Feldstein J, Smith J, et al. 1998. Primary cardiac sarcoma: a novel treatment approach. Chest 114:648-652. Selected Reports.

Oh SY, Yeom SY, Kim KH. 2013. Clinical Implication of Surgical Resection for the Rare Cardiac Tumors Involving Heart and Great Vessels. J Korean Med Sci 28:717-24.

Putnam JB Jr, Sweeney MS, Colon R, et al.1991. Primary cardiac sarcomas. Annals of Thoracic Surgery 51:906-910.

Silverman NA. 1980. Primary cardiac tumor. Ann Surg 191:127-138.

Sturgis EM, Potter BO. 2003. Sarcomas of the head and neck region. Curr Opin Oncol 15: 239-52.

Talbot SM, Taub RN, Keohan ML, et al. 2002. Combined heart and lung transplantation for unresectable primary cardiac sarcoma. J Thorac Cardiovasc Surg 124:1145-1148. 